\title{
La crisis de reproducibilidad de la ciencia y la necesidad de publicar los resultados negativos:
}

En el año 2015 la revista Nature llevó a cabo una encuesta entre científicos de diversas disciplinas, con el objetivo de determinar qué porcentaje de lo publicado en sus respectivos campos consideraban que era reproducible. Sorprendentemente más del $70 \%$ de los encuestados afirmaban haber sido incapaces de replicar el experimento de otro colega y un $60 \%$ no había sido capaces de replicar uno propio. Estas cifras dependían de la disciplina en cuestión eran relativamente bajas en el ámbito de la física y la ingeniería pero muy altas en el ámbito de la medicina y la biología.(1) El origen de esta falta de reproducibilidad es complejo, pero probablemente uno de los principales responsables es el llamado sesgo de publicación.

La probabilidad de que los resultados de una investigación se publiquen debería depender tan solo de su calidad y ser independiente del resultado de la misma. Sin embargo la intuición nos dice que esto no es así. Si hojeamos cualquier revista científica podremos comprobar que cerca del 90\% de los artículos comunican resultados positivos.(2) Ante este hecho sólo caben dos interpretaciones o bien los resultados verdaderos negativos tienden a no publicarse o bien los investigadores están dotados de una intuición extraordinaria y en el 90\% de los casos formulan hipótesis que luego se confirman.

No se trata de una idea nueva. En 1959, ya hace casi seis décadas, Sterling definió por primera vez este sesgo, tras revisar cinco revistas científicas y determinar que el $97 \%$ de los artículos que contenían comunicaban hallazgos positivos.(3) El origen del sesgo de publicación es complejo. En parte podríamos culpar a los directores de las revistas y al proceso editorial que, a sabiendas de que la probabilidad de que un artículo sea citado es más alta si este comunica resultados positivos, los favorecen. (3) Sin embargo numerosos estudios demuestran que los principales responsables del sesgo de publicación son los propios investigadores. Cuando los investigadores no alcanzan la ansiada $p<0,05$ consideran que sus resultados son equivocados 0 
no interesantes y abandonan el proceso de investigación. Por eso este problema también ha recibido la denominación del "file drawer problem". En caso de seguir adelante con la misma, numerosos trabajos afirman que es probable que la investigación termine siendo publicada en una revista de bajo impacto. Algunos trabajos afirman incluso que el grado de significación de los resultados se correlaciona con la precocidad de la publicación (pipeline bias) y el idioma en el que se llevará a cabo la misma. Los resultados positivos se publican antes (especialmente si hacen referencia a tratamientos nuevos y más intervencionistas), pues los directores de la revistas dan prioridad a estos artículos, y lo hacen con mayor probabilidad en revistas de impacto alto, habitualmente en inglés en tanto que los resultados negativos no se publican o se publican tarde y en el mejor de los casos, lo hacen en revistas menos prestigiosas y en lengua no inglesa.(3-6)

Esa selección natural que actúa a distintos niveles y que favorece la publicación de la investigación que arroja resultados positivos hace que el error alfa (esa baja probabilidad de rechazar la hipótesis nula cuando es realmente cierta y que por convenio se ha establecido en el 5\%) llene un porcentaje muy alto de las páginas de nuestras revistas científicas porque la selección natural imperante favorece su publicación. Por el contrario los resultados negativos ciertos (probabilísticamente 1-alfa), permanecerían con una más alta probabilidad abandonados y despreciados en el cajón del investigador (o en el mejor de los casos se publicarían en una revista de bajo impacto) porque el investigador considera que esos resultados no son valiosos.

Por ello toda esta evidencia quedaría fuera del alcance de los meta-análisis. Teóricamente, la metodología sacrosanta de la medicina basada en la evidencia, el meta-análisis, debería estar preparado para subsanar este problema. De hecho se han desarrollado al menos una docena de estrategias para detectar y compensar el sesgo de publicación.(7) Sin embargo, muchos de estos métodos están dotados de una potencia estadística baja y basados en gran medida en la 
subjetividad del investigador.(3) En un trabajo reciente, los autores tras estudiar 300 revisiones y meta-análisis publicados en las 10 revistas de mayor impacto en el ámbitos de la medicina, concluían que en un tercio de los casos los autores ni siquiera intentaban cuantificar el sesgo de publicación, y que un quinto de las mismos contenían importantes sesgos de publicación.(8) Algunos expertos afirman que los autores de un meta-análisis deben estudiar la literatura gris y contactar con los expertos en el tema que estudian para acceder a los resultados no publicados, lo cierto es que esto es muy complicado y raramente se hace. Los resultados verdaderos negativos quedan en muchos casos en poder del investigador inaccesibles a los meta-análisis, y cuando se difunden en muchos casos tampoco se integran en los mismos, porque se publican en revistas no anglosajonas y en muchos casos los autores del metaanálisis incluyen sólo literatura anglosajona en los mismos. Por ello existiría un segundo sesgo de publicación en la trasferencia de los resultados de los artículos originales a los metaanálisis(6) y los resultados negativos están infrarrepresentados en la literatura primaria y muy infrarrepresentados en la secundaria. Para añadir más sesgo al previo, no es raro que cuando una serie ofrece resultados positivos se publique varias veces.(9) La publicación duplicada no siempre lo hace bajo el nombre del mismo autor, por lo que en muchas ocasiones es difícil detectarla. Por poner un ejemplo práctico, es lo que ocurre cuando sucesivas generaciones de residentes utilizan la misma base de datos para realizar su tesis y luego publican sus resultados. Los resultados negativos no llegan a publicarse y los positivos en ocasiones se publican varias veces. El éxito tiene muchos padres pero el fracaso es huérfano y todo esto contribuye a agravar aún más el sesgo. Por eso, los meta-análisis son por definición optimistas e inevitablemente tienden a sobrestimar los efectos de las intervenciones terapéuticas y el peso de los factores de riesgo.

Nos encontramos ante un proceso de selección natural que actúa a distintos niveles y es muy difícil de combatir, porque el investigador busca de forma activa el resultado positivo. Pocas cosas hay más tristes que una tesis sin sus $p$ significativas en las conclusiones. Cuando el 
investigador no encuentra el ansiado resultado, desprecia sus datos y abandona la línea de investigación e inicia otra nueva o en no pocos casos, los cocina elaborando hipótesis posthoc.(10) Esta actitud distorsiona por completo el proceso de investigación y recibe el nombre de HARKing (Hipothesizing After the Results are Known).(11) A nivel de revisión, hay estudios que demuestran que un mismo manuscrito es considerado metodológicamente superior cuando sus resultados son positivos que cuando son negativos.(10) y que los trabajos con resultados negativos que contradicen estudios previos que tuvieron resultados positivos tienen una probabilidad muy baja de ser publicados.(10)

Así que con frecuencia, el hallazgo falso positivo se publica como artículo original en una revista científica, que de este modo lo beatifica (en no pocos casos se trata de un falso positivo y probabilísticamente constituye lo que llamaríamos error alfa). El meta-análisis o la revisión sistemática finalmente acaban por canonizarlo.(12) Una vez santificado el hallazgo en las literatura secundaria pasará a los libros y será muy difícil ponerlo en cuestión.

El problema es complejo porque tampoco es posible publicar todo lo que se investiga. Algunas estrategias como el registro previo de los ensayos clínicos, o la creación de plataformas o revistas que publiquen específicamente resultados negativos pueden ayudar a mitigarlo(12, 13), pero debemos ser conscientes de que en gran medida el origen del sesgo de publicación está en nosotros mismos, en los propios investigadores. Además de la ética y la rigurosidad en el proceso de investigación, es preciso revalorizar el resultado negativo. Este número contiene un interesante artículo publicado por los doctores Nieves-Moreno, Morales Fernández, Domingo Gordo, Maillo, Diaz y Gómez de Liaño, en el que utilizando un riguroso diseño demuestran la escasa eficacia de la brinzolamida en el tratamiento sintomático del nistagmus congénito. El artículo es metodológicamente bueno, pues en él se utiliza el electronistagmógrafo del Dr. Perea y por ello incluye variables objetivas. Además la nula concordancia que encuentran entre exploradores aporta pistas importantes al porque otros 
exploradores han podido encontrar efectos beneficiosos. Confiamos en que en el futuro

contribuya a contrapesar el sesgo de publicación existente en la escasa literatura relacionada.

Sólo felicitar a las autores y confiar en que esta cultura de que publicar los resultados

negativos tiene tanto valor como publicar los resultados positivos se imponga.

\section{Reference List}

1. Baker M. 1,500 scientists lift the lid on reproducibility. Nature. 2016;533(7604):452-4.

2. Matosin N, Frank E, Engel M, Lum JS, Newell KA. Negativity towards negative results: a discussion of the disconnect between scientific worth and scientific culture. Dis Model Mech. 2014;7(2):171-3.

3. Mahid SS, Qadan M, Hornung CA, Galandiuk S. Assessment of publication bias for the surgeon scientist. Br J Surg. 2008;95(8):943-9.

4. Montori VM, Smieja M, Guyatt GH. Publication bias: a brief review for clinicians. Mayo Clin Proc. 2000;75(12):1284-8.

5. Dwan K, Gamble C, Williamson PR, Kirkham JJ, Reporting Bias G. Systematic review of the empirical evidence of study publication bias and outcome reporting bias - an updated review. PLoS One. 2013;8(7):e66844.

6. Carter AO, Griffin GH, Carter TP. A survey identified publication bias in the secondary literature. J Clin Epidemiol. 2006;59(3):241-5.

7. Sutton AJ, Song F, Gilbody SM, Abrams KR. Modelling publication bias in meta-analysis: a review. Stat Methods Med Res. 2000;9(5):421-45.

8. Onishi A, Furukawa TA. Publication bias is underreported in systematic reviews published in high-impact-factor journals: metaepidemiologic study. J Clin Epidemiol. 2014;67(12):1320-6.

9. Tramer MR, Reynolds DJ, Moore RA, McQuay HJ. Impact of covert duplicate publication on meta-analysis: a case study. BMJ. 1997;315(7109):635-40.

10. Thornton A, Lee P. Publication bias in meta-analysis: its causes and consequences. J Clin Epidemiol. 2000;53(2):207-16.

11. Kerr NL. HARKing: hypothesizing after the results are known. Pers Soc Psychol Rev. 1998;2(3):196-217.

12. Nissen SB, Magidson T, Gross K, Bergstrom CT. Publication bias and the canonization of false facts. Elife. 2016;5.

13. Kien C, Nussbaumer B, Thaler KJ, Griebler U, Van Noord MG, Wagner P, et al. Barriers to and facilitators of interventions to counter publication bias: thematic analysis of scholarly articles and stakeholder interviews. BMC Health Serv Res. 2014;14:551.

14. Nieves-Moreno M, L. Morales Fernández L, Domingo Gordo B, Maillo E, Diaz E, Gómez-deLiano R. Brinzolamida tópica en el nistagmo congénito: Estudio retrospectivo. 2017; xxxxxx 